\title{
El impacto económico del
} turismo en localidades pequeñas. Análisis insumoproducto aplicado a los parques Ischigualasto y Talampaya

The Economic Impact of Tourism in Small Towns. Input-Output Analysis Applied to the Ischigualasto and Talampaya Parks

\section{Carlos A. Romero}

CONICET-Universidad de Buenos Aires. Instituto Interdisciplinario de Economía Política de Buenos Aires, Argentina.

carlos.adrian.romero@gmail.com

\section{Juan P. Tarelli}

Escuela de Economía y Negocios, Universidad Nacional de San Martín, Buenos Aires, Argentina. jtarelli@hotmail.com

\section{Lucila Porto}

Facultad de Ciencias Económicas, Universidad de Buenos Aires, Argentina.

lulaporto96@gmail.com 


\section{Resumen}

En el presente artículo se realiza una evaluación del impacto económico de un programa de inversión turística en la región de Valle Fértil-Villa Unión, dentro de las provincias de San Juan y La Rioja (Argentina), utilizando modelos de insumo-producto interregionales. Este tipo de investigaciones regionales presentan como dificultad la escasez de información a nivel subnacional. Para enfrentar este problema, se construyó una matriz de contabilidad social para la región, utilizando un enfoque híbrido. La aplicación de esta metodología se basa en el uso combinado de coeficientes de ubicación y encuestas locales realizadas en los años 2015-2016. Mediante ejercicios de simulación, se obtuvo una correlación positiva entre el incremento de la demanda turística y el crecimiento del producto local. Dicha relación podría ser mayor si se desarrollasen cadenas de valor regionales..

Palabras clave: economía del turismo, economía regional, matrices de contabilidad social regionales, análisis insumo-producto regional.

\section{Abstract}

In this paper, we evaluate the economic impact of a tourism investment program in the Valle Fértil-Villa Unión region, within San Juan and La Rioja provinces (Argentina), through interregional input-output models. This type of regional research presents as a difficulty the lack of information at the subnational level. To solve this issue, we built a social accounting matrix for the region, using a hybrid approach. The application of this methodology was based on the combined use of location coefficients and local surveys carried out within the years 2015-2016. Using simulation exercises, we found a positive correlation between the increase of tourism demand and the growth of the local product. However, this relationship could be higher if regional value chains were developed.

Keywords: : tourism economics, regional economy, regional social accounting matrices, regional input-output analysis.

Journal of Economic Literature (JEL):C67, L83, 022, R15. 


\section{Introducción}

\subsection{Antecedentes y estado del arte}

El objetivo del trabajo fue evaluar el impacto de un programa de inversión turística en la región de Valle Fértil-Villa Unión, dentro de las provincias de San Juan y La Rioja en Argentina. Realizar investigaciones sobre el impacto del turismo presenta la dificultad de no poder separar la población en un grupo de control y otro de tratamiento, dificultando así los análisis econométricos. Si, además, el análisis es a nivel regional, se suma también una restricción relevante: datos deficientes o inexistentes cuyos costos de obtención implicarían la erogación de gran cantidad de recursos. Los modelos de equilibrio general computable (CGE) y modelos de insumo-producto son herramientas conocidas y utilizadas en la literatura de investigación turística. Como antecedentes, se pueden mencionar los estudios de Romero, Mastronardi, Tarelli \& Haslop (2019), Allan, Lecca \& Swales, (2017), Taylor (2010), Taylor, Hardner \& Stewart (2009), Oosterhaven \& Fan (2006), Dwyer, Forsyth \& Spurr (2004), Taylor, Dyer, Stewart, Yunez-Naude \& Ardila (2003), Frechtling \& Horváth (1999) y Sinclair (1998). Con respecto al primero de ellos, Romero \& al. (2019), es uno de los únicos estudios publicados que utiliza técnicas híbridas con el fin de comprender el impacto del turismo en una región (en la provincia de Salta, Argentina).

Pese a ser instrumentos muy utilizados, la implementación de los elementos básicos de los modelos de CCE e insumo-producto (las tablas insumo-producto y las matrices de contabilidad social) requiere más información de la que hay disponible para la mayoría de los departamentos regionales o provinciales sobre estadística/turismo. Esto podría ser un potencial problema para entender cómo el crecimiento de la industria del turismo está cambiando la economía regional. Si las tablas insumo-producto desarrolladas oficialmente no están disponibles, entonces, construir una propia puede ser una solución. Las agencias gubernamentales generalmente desarrollan estas matrices a partir de los datos de encuestas, pero existen opciones más fáciles y económicas como sustitutos de esta metodología. Las técnicas de semiencuestas y sin encuestas no requieren tantos recursos como las técnicas de encuestas y proporcionan resultados confiables y de calidad. En el caso de las técnicas sin encuesta, la metodología del cociente de ubicación (LQ) se puede utilizar como base para la construcción de la tabla insumo-producto. Las estimaciones alcanzadas mediante la aplicación de técnicas LQ pueden reforzarse 
posteriormente con encuestas centradas en ciertas industrias o mercados, bajo un enfoque híbrido.

En este estudio, se construyeron una tabla insumo-producto y una matriz de contabilidad social para la región, utilizando un enfoque híbrido. La aplicación de esta metodología se basó en el uso combinado de coeficientes de ubicación y encuestas locales, recopilándose así datos de hogares y empresas. Estos elementos construidos a medida fueron utilizados en modelos insumo-producto interregional', los cuales permitieron la medición tanto de efectos directos como indirectos, donde se simuló un aumento de la demanda turística. A su vez, con los resultados obtenidos de dichas simulaciones, se implementó un análisis de costo-beneficio y de sensibilidad.

\subsection{Programa de inversión turística}

El programa de inversión turística es un proyecto conjunto del ex Ministerio de Turismo de la Nación Argentina (MINTUR) (actual Ministerio de Turismo y Deportes de la Nación) y el Banco Interamericano de Desarrollo (BID), Ilamado Programa de Desarrollo de Corredores Turísticos ${ }^{2}$ (Programa). Su objetivo es “incrementar el gasto turístico, mejorando el aprovechamiento turístico espacial y temporal de áreas protegidas (APs) y sus destinos asociados, mediante apoyo a la estructuración de productos turísticos, fortalecimiento institucional y mejora de la gestión ambiental" (BID, 2012, p. 42). En este sentido, se financia la generación de un grupo de obras estratégicamente ubicadas, que permitirán la articulación de los corredores en torno a productos/circuitos innovadores, consolidando la gestión en red de las AP y de sus destinos vinculados (BID, 2011 y 2012).

Al focalizar los esfuerzos de inversión en los corredores turísticos, se "minimizan las intervenciones aisladas en atractivos turísticos puntuales $y$, al contrario, se fomenta la coordinación público-privada para mejorar la distribución geográfica de los flujos turísticos" (BID, 2011 p. 10). Para ello, se cuenta con un financiamiento total de 105 millones de dólares y un cronograma de desembolsos de 5 años, donde el BID aporta el $76 \%$ y el gobierno local el $24 \%$ restante.

1 Un modelo abierto de Leontief que incluye solo las transacciones intersectoriales locales, otro modelo cerrado de Leontief que considera endógeno el gasto y los ingresos de los hogares de la localidad y, finalmente, un tercer modelo basado en MCS que incluye, además de los hogares de VF-VU, la participación de los sectores de actividad, tanto de la provincia como del resto de la Argentina.

2 El Programa tuvo como antecedente al Programa de Mejora de la Competitividad del Sector Turismo (1648/OC-AR; 2005) y al Plan Federal Estratégico de Turismo Sustentable 2005-2016 (MINTUR), el cual procuraba promover el turismo en ciertos corredores. Recién a partir del Programa, se incluyó la región Valle Fértil-Villa Unión. 
Dentro de la región bajo análisis se encuentra el corredor homónimo Valle FértilVilla Unión, el cual incluye al parque nacional Talampaya y al parque provincial Ischigualasto.

En lo que sigue, el trabajo se organiza de la siguiente manera: las secciones 2, 3 y 4 introducen los conceptos metodológicos: la sección 2 describe la construcción y estimación de las matrices de contabilidad social y su posterior anidamiento; la sección 3 presenta el modelo de insumo-producto y la sección 4 presenta los ejercicios de simulación, análisis de costo-beneficioy de sensibilidad. La sección 5 expone una breve caracterización de la región y del peso que tiene el turismo en las economías regionales de San Juan y La Rioja. En la sección 6 se analizan los resultados obtenidos. Por último, en la sección 7 se discuten brevemente las conclusiones del trabajo.

\section{Matriz de contabilidad social interregional}

Conceptualmente, una matriz de contabilidad social (MCS) es una representación específica de la economía de un país, región, provincia o localidad durante un periodo determinado. Se trata de una especie de matriz de insumo-producto 3 expandida que incluye, además de la estructura de la producción, datos sobre las interrelaciones de los sectores de producción, los pagos a los factores, la distribución del ingreso y la estructura de demanda de las instituciones locales, así como sus vinculaciones con el exterior. Una MCS se conforma a partir de cuentas de doble entrada; estas registran las transacciones que efectúan los sectores y agentes económicos en un lapso de tiempo específico, generalmente un año. En las filas de la matriz se asientan los ingresos (o ventas) y en las columnas los gastos (o compras). De este modo, el cruce de una fila con una columna permite identificar las cuentas involucradas en una transacción específica. Las MCS, además de ser una herramienta fundamental para entender la circularidad de los flujos de una economía, son el primer paso a la hora de la medición de impacto de un proyecto sobre un área objetiva. Una MCS configura la base de información sobre la que se construyen modelos de simulación (Miller \& Blair, 2009).

Para este trabajo se construyó una MCS (anidada) con desagregación en tres regiones: 1) local, correspondiente a las transacciones de Valle Fértil y Villa Unión (VFVU); 2) regional, con las transacciones de las demás regiones de las provincias San Juan y La Rioja exceptuando VF-VU (SJ-LR) y 3) nacional, que representa las transacciones del resto de Argentina (R-ARG) exceptuando las provincias de SJ-LR. La utilización de una matriz anidada permite captar las vinculaciones dentro de cada región en particular y de ellas con las restantes regiones.

3 Una matriz insumo-producto es una representación de las interrelaciones entre los sectores de una economía. Matemáticamente se expresa como un conjunto de ecuaciones lineales, donde cada una describe la distribución del producto sectorial en toda la economía (Miller \& Blair, 2009). 
En la tabla1 puede verse la estructura de la matriz. En la columna sombreada se representan los gastos de las industrias, los hogaresyel resto de la demanda final local $\mathbf{4}$ de VF-VU: 1) dentro de la región (transacciones locales), 2) en el resto de las provincias (gastos en la región) y 3) en el resto de la Argentina y las importaciones del resto del mundo (gastos fuera de la región y RM). Similarmente, la fila sombreada representa los ingresos de las industrias y los factores productivos dentro y fuera de la región. A modo de ejemplo, los ingresos de los hogares correspondientes a salarios pagados por el gobierno nacional a trabajadores domiciliados en las localidades, está captado en "ingresos fuera de la región”.

Tabla 1. Estructura de la MCS de Valle Fértil-Villa Unión

\begin{tabular}{|c|c|c|c|c|}
\hline & & & & \\
\hline & & VF-VU & Resto SJ-LR & Resto Argentina \\
\hline & & 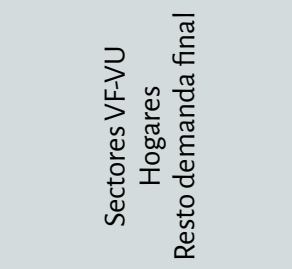 & 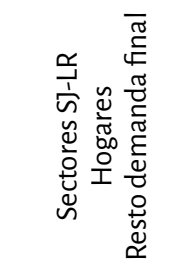 & 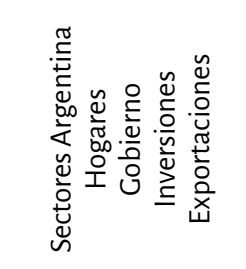 \\
\hline VF-VU & $\begin{array}{l}\text { Sectores productivos } \\
\text { Valor agregado }\end{array}$ & Transacciones locales & & $\begin{array}{c}\text { Ingresos fuera de la } \\
\text { región }\end{array}$ \\
\hline SJ-LR & $\begin{array}{l}\text { Sectores productivos } \\
\text { Valor agregado }\end{array}$ & Gastos en la región & $\begin{array}{c}\text { Transacciones } \\
\text { Regionales }\end{array}$ & \\
\hline R-ARG & $\begin{array}{l}\text { Sectores productivos } \\
\text { Valor agregado } \\
\text { Importaciones }\end{array}$ & $\begin{array}{l}\text { Gastos fuera de la } \\
\text { región y RM }\end{array}$ & & $\begin{array}{l}\text { Transacciones resto } \\
\text { Argentina y con RM }\end{array}$ \\
\hline
\end{tabular}

Nota: VF-VU: Valle Fértil y Villa Unión; SJ-LR: resto de las Provincias de San Juan y La Rioja; R-ARG: resto de la Argentina; RM: resto del Mundo. Fuente: elaboración propia.

Para la estimación de la MCS se utilizaron métodos directos e indirectos (Greenstreet, 1989; Szabó, 2015). Con tal fin, se llevaron a cabo encuestas presenciales a empresas y hogares de las localidades de Valle Fértil y Villa Unión, haciendo un mayor énfasis en el área del turismo. Esto posibilitó un mayor nivel de detalle en la estimación de la MCS local. Para la matriz regional San Juan-La Rioja se adoptaron métodos indirectos de estimación. Para la Argentina, se utilizó la correspondiente al año 2012 (Coremberg, Romero, Mastronardi \& Vila Martínez, 2016).

4 El principal componente del resto de la demanda final local es el gasto de los gobiernos de ambas localidades. 


\subsection{Estimación de la MCS de VF-VU}

En relación a la base de datos microeconómicos, se tomó la encuesta realizada a hogares y empresas por Macroconsulting en los años 2015-2016.

Respecto a la metodología seguida en la construcción de la muestra de empresas, no solo estaban incluidas las empresas turísticas más representativas, sino también una muestra aleatoria de empresas, ajenas a dicha rama, pero con un peso significativo para la región. En cuanto al cuestionario propiamente dicho, las preguntas apuntaban, entre otras cuestiones, al monto total de ventas según área de origen del consumidor y tipo de agente (final, intermedio, gobierno y resto del mundo) y al total de las compras intermedias realizadas según producto (clasificadas según el origen regional de las mismas). Además de esta información, se registraron datos en materia impositiva y salarial, así como el valor bruto de producción. Con el fin de lograr una base de datos más precisa, se utilizó un cuestionario específico para cada rama de actividad (Macroconsulting, 2016).

La MCS global cuenta con 12 sectores productivos que se desarrollan en el conglomerado de las localidades. En cuanto a la producción fuera de la localidad, se consideró un sólo sector productivo agregado.

Para la construcción de la muestra de hogares, Macroconsulting (2016) partió de la cartografía elaborada por el INDEC para el Censo de Población, Hogares y Viviendas realizado en el año 2010. A partir de ella, definió los radios censales. Cada radio se consideró como un estrato y a cada uno de ellos se le asignó una cantidad de casos, proporcional a su participación en el total de viviendas. El relevamiento estuvo destinado a 450 hogares y, en términos generales, se relevó información relacionada al consumo de cada uno de ellos según producto y fuente de ingreso, dividiendo por tipo de actividad y región donde se realiza.

\subsection{Estimación de las MCS Regionales}

A causa de la escasez de datos a nivel subnacional, para las MCS de fuera de la localidad se tomó la matriz de contabilidad social nacional y se la utilizó como base para su regionalización. Debido a que las fuentes secundarias no estaban expresadas en los años ni con el nivel de desagregación deseados, fue necesario recurrir a métodos indirectos (Flegg, Webber \& Elliott,1995; Flegg \& Webber, 2000; Flegg, Mastronardi \& Romero, 2015) y a métodos híbridos (Mastronardi, 2015; Mastronardi \& Romero, 2012; Chisari, Mastronardi \& Romero, 2012) de estimación.

Respecto a los primeros métodos de estimación, se siguió la metodología propuesta por Flegg \& Webber (1997). Ella parte del supuesto planteado por Jensen, Mandeville \& Karunaratne (1979), según el cual los coeficientes intrarregionales $\left(a_{i j}{ }^{R}\right)$ difieren de los coeficientes técnicos nacionales $\left(a_{i j}{ }^{N}\right)$ solo por un factor de participación en el comercio regional $\left(l q_{i j}\right)$. Matemáticamente: $a_{i j}{ }^{R}=l q_{i j} a_{i j}{ }^{N}$. El 
otro supuesto que se adopta es que la tecnología en la región es similar a la media del país. Esto permite, sobre la base del LQ elegido, distinguir dentro de la región entre sectores que son autosuficientes y carecen de importaciones regionales y aquellos que no lo son.

El factor de participación en el comercio regional $\left(l q_{i j}\right)$ fue estimado, entonces, a partir de la fórmula FLQ (Flegg Location Quotient) que postula una relación inversa entre el tamaño de la región y la propensión a importar de otras regiones.

$$
F L Q_{i j}=\frac{P B G_{i, R} / P B G_{i, N}}{P B G_{j, R} / P B G_{j, N}} \times \lambda^{*} \text { donde } \lambda=\left[\log _{2}\left(1+\frac{P B G_{R}}{P B G_{N}}\right]^{\delta}, 0 \leq \delta \leq 1\right.
$$

Los PBC hacen referencia a los productos brutos geográficos a nivel regional $(R)$ y a nivel nacional $(N)$; $\lambda$ es un factor que pondera el tamaño (importancia) de la región en el país, expresado en el ratio entre el PBC regional respecto del nacional. La idea detrás de dicha inclusión es que las regiones más grandes ajustarán en una menor proporción el coeficiente nacional, ya que se cree que estas importan relativamente menos que las más chicas. Además, lambda depende también de $\delta$, el cual está vinculado con las importaciones interregionales, cuanto más se acerque a uno este parámetro, mayores serán las importaciones interregionales.

Respecto a los métodos híbridos, se empleó el método RAS y el de entropía cruzada $^{5}$. El primero, también conocido como método de ajuste biproporcional, es un proceso iterativo que requiere conocer los totales fila y columna para realizar un ajuste de una matriz de partida (Bacharach, 1970). El método de entropía cruzada es un método de optimización que busca minimizar una medida de distancia entre una matriz de partida y diferentes matrices calculadas que cumplan con una determinada cantidad de restricciones -tanto tecnológicas como transaccionales(Robinson, Cattaneo \& El-Said, 2001).

\subsection{Anidamiento de las MCS}

Una vez estimadas las tres MCS (local, provincial y del resto de Argentina) se siguió con su anidamiento. Los totales columna de cada uno de los sectores representan su el valor bruto de producción (VBP) al contabilizar los impuestos. Fueron contemplados dos factores que componen el valor agregado (trabajo y capital) y tres tipos de impuestos en función del gobierno que los recauda (impuestos municipales, provinciales y nacionales). Se han considerado 3 cuentas endógenas ${ }^{6}$ de hogares de

5 Para un mayor desarrollo de dichos métodos ver McDougall, (1999).

6 El consumo de los hogares se estima sobre la base del cierre macroeconómico, en conjunto con la demanda del resto de Argentina, con el objetivo de asegurar la consistencia interna de la matriz. 
acuerdo a la residencia: representativo del conglomerado de localidades de Valle Fértil y Villa Unión; representativo del resto de San Juan y La Rioja y representativo del resto de Argentina. Por otra parte, el gasto de los turistas en las localidades se desagregó según su nacionalidad (argentinos y extranjeros).

\section{El modelo}

Una vez obtenida la matriz de contabilidad social interregional, esta fue utilizada como base para los modelos de insumo-producto (MIP). La idea detrás de la utilización de estos modelos es la estimación de los multiplicadores. Estos miden los efectos sobre una economía en términos de cambios en la producción y el valor agregado a nivel sectorial, ante un cambio exógeno, comparando los valores iniciales respecto de los finales (Miller \& Blair, 2009). Los efectos totales que se desprenden de dicho shock son de tipo directo, indirecto e inducido. "Los efectos directos son aquellos pertenecientes al sector que se ve directamente afectado por el shock" (Breisinger, Thomas \& Thurlow, 2009, p.13). Los efectos indirectos son los que se evidencian luego de que el sector afectado se interrelaciona con los otros sectores de la economía a través de su producción y consumo (Breisinger \& al., 2009). Por último, los inducidos son aquellos generados por el mayor ingreso que perciben los hogares por ser oferentes del factor trabajo (Miller \& Blair, 2009). En lo que respecta a este trabajo, los ejercicios de simulación de un aumento de la demanda turística constituyen el cambio exógeno a evaluar.

Para la construcción del modelo de insumo-producto interregional, se siguió la notación presente en Miller \& Blair (2009). Dada una matriz ${ }^{\mathbf{7}}$ del tipo:

$Z=\left[\begin{array}{ll}Z^{R R} & Z^{R N} \\ Z^{N R} & Z^{N N}\end{array}\right]$

Donde $Z^{R R}$ tanto como $Z^{N N}$ representan las matrices de transacciones, por un lado, entre aquellos sectores que pertenecen a la región $R y$, por otro, entre aquellos sectores que pertenecen al resto del país $(N)$. A su vez, la matriz $Z^{R N}$ contempla aquellas ventas de sectores de la región a sectores del resto del país y $Z^{R N}$ contabiliza las compras de los sectores de la región a sectores del resto del país. Nótese que las matrices ubicadas en los extremos superior derecho e inferior izquierdo pueden entenderse respectivamente como exportaciones e importaciones interregionales.

Se puede formular, entonces, una ecuación que resuma el destino de las ventas totales de un sector determinado de una cierta región en una ecuación de la forma:

7 Si bien para el caso de Valle Fértil-Villa Unión el total de las regiones a considerar es de 3 (local, provincial y nacional), aquí se introduce un modelo de 2 regiones (local, nacional) dado que su generalización a 1 de 3 regiones es sencilla y exponerlo de esta manera facilita su comprensión. 
$x_{i}^{R}=Z_{i j}^{R R}+Z_{i j}^{R N}+f_{i}^{R}$

Donde $x_{i} R$ representa las ventas totales de las firmas del sector $i$ de la región $R$; $Z_{i j}{ }^{R R}$ y $Z_{i j}^{R N}$ son las ventas totales de las firmas del sector $i$ a las firmas del sector $j$ de la región $R$ y del resto del país, respectivamente; $y f_{i}^{R}$, el total de las ventas de firmas del sector $i$ dedicadas a consumo final, realizado tanto dentro como fuera de la región.

A partir del ratio entre los componentes de la matriz (2) y el resultado de la ecuación (3), puede obtenerse el conjunto de coeficientes técnicos tanto para el comercio intrarregional $\left(a_{i j}{ }^{R R} ; a_{i j}{ }^{N N}\right)$ como para el interregional $\left(a_{i j}{ }^{R N} ; a_{i j}{ }^{N R}\right)$.

$a_{i j}^{R R}=\frac{Z_{i j}{ }^{R R}}{x_{j}^{R}} ; a_{i j}{ }^{N N}=\frac{Z_{i j}{ }^{N N}}{x_{j}^{N}} ; a_{i j}^{R N}=\frac{Z_{i j}{ }^{R N}}{x_{j}^{N}} ; a_{i j}{ }^{N R}=\frac{Z_{i j}{ }^{N R}}{x_{j}^{R}}$

Estos coeficientes técnicos se calculan como el cociente entre las compras del sector $j$ al sector $i$, con respecto al valor de producción del sector $j$, para cada una de las regiones. Se puede, de esta manera, reexpresar la ecuación (3) en términos de coeficientes interregionales:

$x_{i}^{R}=a_{i j}^{R R} X_{j}^{R}+a_{i j}^{R N} X_{j}^{N}+f_{i}^{R}$

Sobre la base de la ecuación (5), es posible plantear dos ecuaciones que permiten comprender las interacciones que se generan dentro de cada región como así también las que se dan entre ellas:

$\left(I-A^{R R)}{ }_{X}^{R}-A^{R N} X^{N}=f^{R}\right.$

$-A^{N R} X^{R}+\left(I-A^{N N}\right) x^{N}=f^{N}$

donde las submatrices $A$ son

$A^{R R}=\left[\begin{array}{ccc}a_{11}^{R R} & \cdots & a_{1 j}^{R R} \\ \vdots & \ddots & \vdots \\ a_{j 1}^{R R} & \cdots & a_{j j}^{R R}\end{array}\right], \quad A^{R N}=\left[\begin{array}{ccc}a_{11}^{R N} & \cdots & a_{1 j}^{R N} \\ \vdots & \ddots & \vdots \\ a_{j 1}^{R N} & \cdots & a_{j j}^{R N}\end{array}\right]$

Dadas las expresiones introducidas hasta ahora, se puede reexpresar el comercio interregional e intrarregional de la región $R$ y del resto del país ( $N$ ) bajo una misma ecuación:

$(I-A) x=f$ 
siendo cada uno de los vectores y matrices que la componen

$A=\left[\begin{array}{ll}A^{R R} & A^{R N} \\ A^{N R} & A^{N N}\end{array}\right], x=\left[\begin{array}{l}x^{R} \\ X^{N}\end{array}\right], f=\left[\begin{array}{l}f^{R} \\ f^{N}\end{array}\right], I=\left[\begin{array}{ll}I & 0 \\ 0 & I\end{array}\right]$

Para poder observar cómo el modelo captura las variaciones generadas por shocks exógenos, puede pensarse a las variables $x^{R} ; x^{N} ; f^{R}$ y $f^{R}$ de las ecuaciones (6) y (7) no como valores absolutos, sino como variaciones: ${ }^{8}$ y $\Delta x^{R} ; \Delta x^{N} ; \Delta f^{R}$ y $\Delta f^{R}$. Además, se asumirá por simplicidad que $\Delta f^{N}=0$. Bajo estas suposiciones, puede resolverse la ecuación (5) para $x^{N}$ :

$X^{N}=\left(I-A^{N N}\right)^{-1} A^{N R} X^{R}$

y reemplazando en la ecuación (6):

$\left(I-A^{R R}\right) x^{R}-A^{R N}\left(I-A^{N N}\right)^{-1} A^{N R} x^{R}=f^{R}$

Nótese que si la ecuación (12) hiciera referencia a un modelo de una única región, su forma sería igual al primer término de la ecuación (9):

$\left(I-A^{R R}\right) x^{R}=f^{R}$

lo que implica que el segundo término de la ecuación (12):

$A^{R N}\left(I-A^{N N}\right)^{-1} A^{N R} X^{R}$

introduce las dinámicas interregionales en el modelo. Puede verse que, por un lado, $A^{N R} X^{R}$ contempla aquellas compras que la región $R$ realiza al resto del país $(N)$ para sustentar el aumento en el producto. Por otro lado, $A^{R N}\left(I-A^{N N}\right)^{-1}$ representa los efectos directos e indirectos que tendrá los aumento en la demanda para el resto del país.

\section{Simulaciones, análisis costo-beneficio y de sensibilidad}

Se simula un aumento de la demanda turística de $10 \%$. En la práctica, este shock puede estar influenciado por diferentes razones, como ser campañas de promoción locales o nacionales, desarrollo de infraestructura que faciliten el acceso a los parques (por ejemplo, mejoras de las rutas, accesos, terminales aeroportuarias, etc.), el tipo de cambio, entre otros.

8 Esto surge como consecuencia de la linealidad del MIP, que provoca que el modelo arroje resultados similares en niveles y en variaciones (Miller \& Blair, 2009). 
El aumento de la demanda del turismo es modelado mediante un incremento del gasto total turístico del hogar representativo de cada región (VF-VU, SJ-LR, R-ARG). A su vez, la demanda se divide en turismo doméstico y receptivo (internacional). Los resultados obtenidos por técnicas insumo-producto se pueden utilizar para responder preguntas relacionadas con otras variables de interés. Así, pueden contribuir al análisis costo-beneficio de diversas políticas turísticas (inversiones en promoción o infraestructura) y evaluar el monto de beneficios requeridos para compensar los costos de la política analizada (Taylor, 2010).

Se emplea un conjunto de tres MIP con las siguientes especificaciones:

- M1: modelo abierto de Leontief que incluye solo las transacciones intersectoriales locales.

- M2: modelo cerrado de Leontief que considera endógeno el gasto y los ingresos de los hogares de la localidad.

- M3: modelo basado en MCS que incluye, además de los hogares de VF-VU, la participación de los sectores de actividad, tanto de la provincia como del resto de la Argentina.

La utilización de modelos interregionales permite capturar, además de los efectos directos e indirectos propios de un modelo insumo-producto, los efectos entre las distintas regiones. Dado que un aumento de la demanda en una región tiene impacto tanto en su región como en otras, se puede pensar a esos efectos intersectoriales como fugas del impulso de la demanda. Esto se debe a que parte del aumento de la producción necesario para satisfacerla se cubre con importaciones de otras regiones, distintas a aquella donde se originó el incremento de la demanda. En este sentido, unos mayores coeficientes dentro de la matriz local permitirían, a través de un mayor encadenamiento local, extender el impacto sobre la economía regional (Miller \& Blair, 2009).

En el análisis costo-beneficio se sigue la metodología de Taylor (2010). ${ }^{9}$ Este tipo de ejercicios se propone determinar ex ante si un determinado plan de inversión va a ser o no redituable a partir de la evaluación de su valor actual neto (VAN). Aplicándolo al caso de estudio, se intenta evaluar el préstamo otorgado por el BID para realizar inversiones en el corredor Ischigualasto-Talampaya.

Con los costos y beneficios es posible calcular el valor actual neto (VAN) del proyecto:

$V A N=\sum_{T=0}^{T}\left(\frac{Y_{t}^{p}-Y_{t}^{s p}}{(1+r)^{t}}-I_{t}\right)$

9 En la misma línea de análisis se encuentra el artículo de Banerjee, Cicowiez, \& Gachot (2015). 
donde $t$ es un subíndice que hace referencia al periodo de tiempo; $T$ es la cantidad de años definidos para el análisis (20 años); y $Y_{t}^{p} Y_{t}^{s p}$ representan los beneficios con y sin el proyecto, respectivamente; $r$, la tasa de interés asumida (12\%); e $I$, el costo del proyecto en el momento $t$ (USD $6904000^{10}$, monto tomado como un costo inicial y único). Los beneficios derivados de la inversión son el resultado del aumento de visitantes a los parques (aumento de una única vez). Se supone que la inversión realizada tiene efecto sobre el turismo a partir del año siguiente. El valor de la variable $Y_{t}^{p}$ se obtuvo a partir de los MIP en los ejercicios de simulación (que consideran un aumento del $10 \%$ del gasto total de los turistas). ${ }^{\mathbf{1 1}}$

El análisis de sensibilidad consiste en variar la tasa de expansión del gasto turístico para observar a qué punto el VAN obtenido del flujo de fondos se vuelve nulo.

\section{La región bajo estudio}

\subsection{Breve caracterización de la región bajo estudio}

El parque provincial Ischigualasto está ubicado al noreste de la provincia de San Juan. Por ruta, se encuentra aproximadamente a unos $300 \mathrm{~km}$ de la ciudad capital y a 70 km de San Agustín del Valle Fértil, siendo esta la última localidad que cuenta con los servicios y el equipamiento necesarios para el turista a la hora del ingreso al parque. ${ }^{12}$ Por otro lado, en la provincia contigua, La Rioja, se encuentra el parque nacional Talampaya, el cual se ubica a alrededor de $200 \mathrm{~km}$ de la capital provincial. Su principal centro de servicios y apoyo es la ciudad de Villa Unión (a 50 km).

Ambos parques, en el año 2000, fueron reconocidos internacionalmente como patrimonio natural mundial por la UNESCO. ${ }^{13}$ La puesta en valor, la protección y el reconocimiento del parque provincial, tiene su origen en el interés científico, principalmente, de las ramas de la paleontología, la geología y la arqueología'14 (Salvadeo \& Ruarte, 2018).

10 "Programa de desarrollo de corredores turísticos" (préstamo del BID N²606 / OC-AR).

11 El horizonte temporal y la tasa de interés elegidas se basan en las guías de evaluación de proyectos del BID (Taylor, 2010).

12 Es por ello, que, pese a que la ciudad Los Baldecitos esté ubicada más cerca, no fue elegida como la sede para el relevamiento a los hogares.

13 UNESCO, Ischigualasto / Talampaya Natural Parks. https://whc.unesco.org/en/list/966

14 Los parques tienen una riqueza excepcional en cuanto a recursos arqueológicos, geológicos y paleontológicos. De hecho, allí se descubrieron los restos fósiles de los dinosaurios más primitivos de los que se tiene registro (Rariza, 2015). Su atractivo geológico tiene origen en un choque de placas producido hace 40 millones de años entre la placa de Nazca y la placa Continental, quedando así Ischigualasto como el único lugar en el mundo donde se encuentra una secuencia completa, y totalmente expuesta, de sedimentos continentales del período triásico (Alcober, Martínez \& Colombi, 2008). Sumado a ello, también existen pinturas de arte rupestre realizadas por los pueblos originarios precolombinos. 
Ambas AP forman parte del corredor turístico Valle Fértil-Villa Unión. Conceptualmente, "los corredores turísticos son las vías de conexión entre las zonas, las áreas, los complejos, los centros, los conjuntos, los atractivos turísticos, los puertos de entrada del turismo receptivo y las plazas emisoras del turismo interno, que funcionan como el elemento estructurador del espacio turístico" (Boullón, 1997, p. 80-81).

En la figura 1 puede observarse la ubicación geográfica de los parques.

\section{Figura 1. Ischigualasto y Talampaya. Corredor Valle Fértil-Villa Unión}

\section{Ischigualasto \\ Parque Provincial}

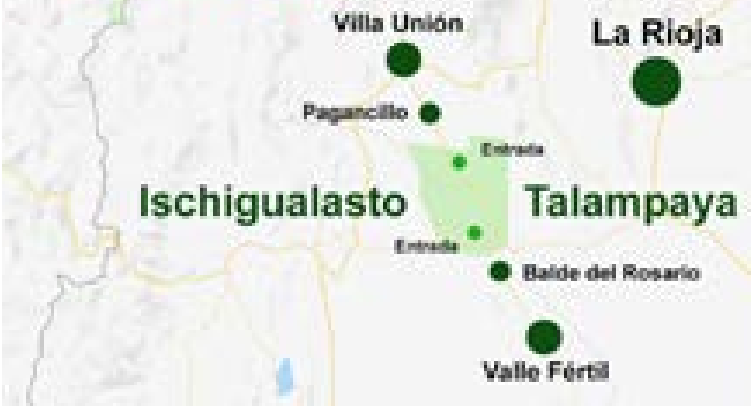

San Juan

Talampaya

Parque Nacional

Fuente: Mitrovic, M. (2019). https://happyfrogtravels.com/es/valle-fertil-talampaya-e-ischigualasto/

En términos de extensión, ambos parques abarcan 278 mil hectáreas, aproximadamente, de las cuales el $23 \%$ corresponde a Ischigualasto y el $77 \%$, a Talampaya (Vergara, 2012).

\subsection{Importancia del turismo en la región}

Según las estadísticas provisionales de la Organización Mundial del Turismo ${ }^{15}$, Argentina fue el país más visitado de América del Sur en 2018, con aproximadamente 6,9 millones de llegadas internacionales. Este desempeño permitió al sector turístico representar el $37 \%$ de las exportaciones de servicios (siendo el segundo mayor exportador de servicios del país) y el 7,6\% de las exportaciones totales (5.800 millones de dólares) ${ }^{\mathbf{1 6}}$. Asimismo, de acuerdo con estadísticas propias elaboradas

15 Fuente: World Tourism Organization (2020), Country Profile-Inbound Tourism.

https://www.unwto.org/country-profile-inbound-tourism

16 Estadísticas obtenidas a partir de Indec (2018), Balanza de pagos, posición de inversión internacional y deuda externa. https://www.indec.gob.ar/indec/web/Nivel4-Tema-3-35-45 
en base a la Encuesta de Viaje y Turismo de los Hogares (EVyTH) ${ }^{\mathbf{1 7}}$ de Argentina, en 2018, el turismo interno alcanzó los 45,5 millones de viajes, generando un gasto total de 7.000 millones de dólares.

En particular, las provincias de La Rioja y San Juan explican conjuntamente alrededor de un millón de viajes internos anuales, ${ }^{\mathbf{1 8}}$ lo que representa el 2,2 \% del turismo interno del país. La importancia de estas dos provincias en conjunto también puede ser analizada a partir de la ocupación hotelera. En efecto, de acuerdo con la Encuesta de Ocupación Hotelera, ambas provincias explican el 0,7 \% y el $1 \%$ del total de las pernoctaciones y de los viajeros del país, respectivamente (considerando tanto pernoctes/viajeros residentes como no residentes).

Pese a ser el menor en tamaño, es Ischigualasto quien atrae una mayor cantidad de visitantes. En efecto, en el año 2018, tuvo 81 mil visitantes, mientras que Talampaya rondó los 60 mil. Sin embargo, es bajo el porcentaje de visitantes anuales de estos parques respecto del total de los parques nacionales (PN) que reciben el conjunto de AP más relevantes del país, ya que ninguno de ellos supera el 2,5\% (figura 2).

Figura 2. Evolución de los visitantes a los parques Talampaya e Ischigualasto (eje izquierdo) y a los parques nacionales de la Argentina (eje derecho) (2009-2018)

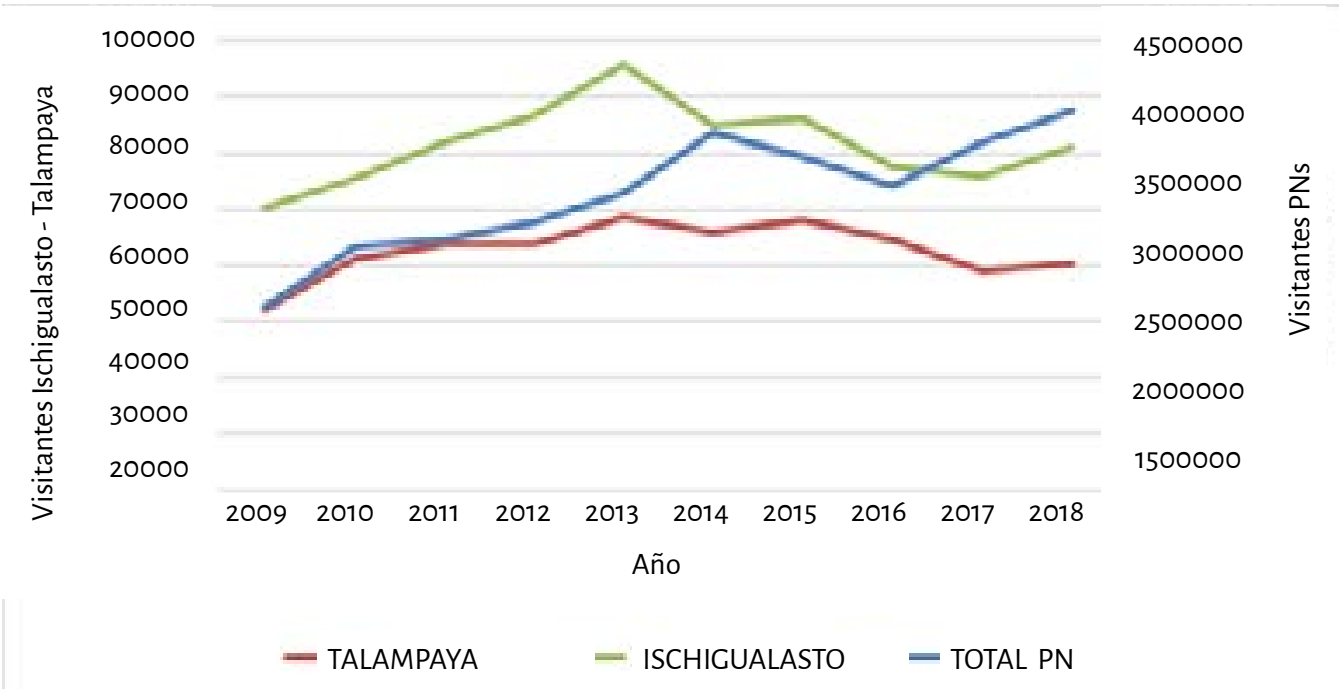

Fuente: elaboración propia basada en datos del Ministerio de Turismo y Deportes de la Nación (http://www.yvera.tur.ar/estadistica/info/parques-nacionales/informes-tecnicos).

Nota: la serie Total PN incluye 35 áreas protegidas que representan aproximadamente un $80 \%$ del total de los PN del país.

17 La EVyTH es una encuesta telefónica realizada por el Ministerio de Turismo de la Nación en la que se relevan, mensualmente, 2.500 hogares pertenecientes a 32 aglomerados (los mismos que son considerados por la Encuesta Permanente de Hogares), distribuidos a lo largo del país. A partir de esta fuente de información, el MINITUR estima la cantidad de viajes que realizan los residentes argentinos por su país.

18 Cantidad de viajes internos que reciben ambas provincias en conjunto. Promedio anual para los años comprendidos en el período 2012-2018. Elaboración propia en base a EVyTH 2018. 


\section{Resultados}

\subsection{Datos obtenidos a través de las encuestas a hogares y a empresas}

A partir de los relevamientos realizados a las empresas de la región, fue posible la desagregación en 12 sectores productivos. Dichos sectores se desarrollan en el conglomerado de las localidades Valle Fértil-Villa Unión y representan el 0.28 \% del VA de San Juan-La Rioja. Los datos se presentan en la tabla 2.

Tabla 2. VF-VU, 2015. Participación sectorial en el PBI local y porcentaje de compras y ventas fuera de la localidad

\begin{tabular}{|lccc|} 
Sectores & Estructura VAB & $\begin{array}{c}\text { Compras fuera de } \\
\text { la localidad }\end{array}$ & $\begin{array}{c}\text { Ventas fuera de la } \\
\text { localidad }\end{array}$ \\
\hline Sector Primario & $5.9 \%$ & $8.3 \%$ & $67.3 \%$ \\
Alimentos, Bebidas y Tabaco & $0.4 \%$ & $43.4 \%$ & $14.6 \%$ \\
Textiles, Cuero y Madera & $0.6 \%$ & $37.3 \%$ & $0.0 \%$ \\
Resto de Industria & $0.3 \%$ & $98.1 \%$ & $45.2 \%$ \\
Construcción & $10.9 \%$ & $100.0 \%$ & $0.0 \%$ \\
Comercio & $8.8 \%$ & $89.5 \%$ & $16.0 \%$ \\
Hoteles & $9.8 \%$ & $80.9 \%$ & $96.0 \%$ \\
Restaurantes & $3.6 \%$ & $50.0 \%$ & $66.5 \%$ \\
Transporte terrestre yacuático & $1.3 \%$ & $69.4 \%$ & $74.8 \%$ \\
Agencias y Transporte aéreo & $8.5 \%$ & $61.0 \%$ & $99.7 \%$ \\
Otros servicios turísticos & $0.3 \%$ & $96.5 \%$ & $99.5 \%$ \\
\hline Otros servicios & $49.6 \%$ & $100.0 \%$ & $76.5 \%$ \\
\hline Total & $100.0 \%$ & $81.8 \%$ & $69.5 \%$ \\
\hline
\end{tabular}

Fuente: elaboración propia basada en datos de Macroconsulting (2016).

Con respecto a la encuesta a hogares, para citar un ejemplo de los resultados haIlados, en relación a la estructura de gasto de los hogares, se estimó que, del ingreso total recibido por ocupaciones, se destina (en promedio) más de un $50 \%$ a la compra de Alimentos y bebidas; el $20 \%$ a salud y el $2.6 \%$ a educación. Por otro lado, en el ámbito laboral, el 10.2 \% de los hogares se desempeñan en actividades económicas relacionadas al turismo, siendo el rubro alojamiento el mayoritario (4.1\%). No obstante, si se consideran todas las actividades, los servicios de enseñanza, salud y seguridad absorben la mayor cantidad de empleo (37.5\%).

En relación al componente turístico, la tabla 3 resume los datos estimados sobre la estructura del gasto promedio diario de los turistas. 
Tabla 3. Estructura del gasto promedio diario de los turistas desagregado por tipo de turismo para el corredor Valle Fértil-Villa Unión. EVyTH 2012-2015 y EVyTH 2006

\begin{tabular}{lcc|}
\hline Concepto & Interno & Receptivo \\
\hline Alojamiento & $37.7 \%$ & $30.1 \%$ \\
Alimentación, & $41.1 \%$ & $30.2 \%$ \\
cultura, recreación y excursiones & $8.4 \%$ & $9.1 \%$ \\
Compra de bienes & $7.6 \%$ & $11.4 \%$ \\
Otros servicios & $5.0 \%$ & $19.2 \%$ \\
Total localidad & $76.1 \%$ & $69.7 \%$ \\
Transporte & $23.9 \%$ & $30.3 \%$ \\
\hline Total Viaje & $100.0 \%$ & $100.0 \%$ \\
\hline
\end{tabular}

Nota: la desagregación del turismo en interno y receptivo hace referencia al lugar de residencia, es decir, si pertenece a las provincias del corredor o si es de otras provincias.

Fuente: elaboración propia en base a la EVyTH 2012-2015 (datos) y EVyTH 2006 (estructura de gasto); la EOH y registros administrativos.

En la tabla 4 se presenta una versión simplificada de la matriz de contabilidad social anidada estimada. Por una cuestión de presentación, se muestra con tres sectores agregados de sectores productivos: primario, industria y servicios.

Tabla 4. Versión simplificada de la MCS anidada (miles de ARS a valor de 2014)

\begin{tabular}{|c|c|c|c|c|c|c|c|c|}
\hline & Primario & Industria & Servicios & $\begin{array}{c}\text { Sectores } \\
\text { S]-LR }\end{array}$ & $\begin{array}{l}\text { Sectores resto } \\
\text { de Argentina }\end{array}$ & $\begin{array}{l}\text { Hogar } \\
\text { VF-VU }\end{array}$ & $\begin{array}{c}\text { Resto } \\
\text { demanda } \\
\text { final }\end{array}$ & Totales \\
\hline Primario & 4,446 & 14 & 198 & & 10,885 & 621 & & 16,164 \\
\hline Industria & 43 & 42 & 1,284 & & & 1,724 & 973 & 4,065 \\
\hline Servicios & 580 & 191 & 10,879 & 37,952 & 8,461 & 24,894 & 176,296 & 259,253 \\
\hline Sectores SJ-LR & 456 & 1,442 & 72,337 & $14,268,144$ & $16,384,090$ & 61,340 & $91,579,299$ & $122,367,108$ \\
\hline $\begin{array}{l}\text { Sectores resto } \\
\text { de Argentina }\end{array}$ & 2 & 2 & 6,093 & $34,684,761$ & $3,208,868,669$ & 95,859 & $5,012,141,355$ & $8,255,796,741$ \\
\hline Trabajo & 6,626 & 671 & 84,728 & $16,898,560$ & $1,310,233,379$ & & & \\
\hline Capital & 1,447 & 1,241 & 38,420 & $31,018,289$ & $1,820,283,808$ & & & \\
\hline Impuestos & 2,564 & 461 & 45,315 & $17,951,753$ & $1,482,817,223$ & & & \\
\hline $\begin{array}{l}\text { Resto del } \\
\text { mundo }\end{array}$ & 0 & & & $7,507,649$ & $417,190,225$ & & & \\
\hline Totales & 16,164 & 4,065 & 259,253 & $122,367,108$ & $8,255,796,741$ & & & \\
\hline
\end{tabular}

Fuente: elaboración propia en base a información primaria y secundaria mencionada en el texto. 


\subsection{Resultados obtenidos a partir de los ejercicios de simulación}

A continuación, se presentan los resultados obtenidos con los modelos de simulación. Todos están expresados en miles de pesos (ARS) a valor de 2014.

Tabla 5. Impacto total sobre la producción

\begin{tabular}{|lcccccc|} 
Efecto & \multicolumn{2}{c}{ M1 } & \multicolumn{2}{c}{ M2 } & \multicolumn{2}{c|}{ M3 } \\
& $\$$ & Mult. & $\$$ & Mult. & $\$$ & Mult \\
\hline Directo & 6,258 & 1.000 & 6,258 & 1.000 & 6,258 & 1.000 \\
\hline Indirecto & 926 & 0.148 & 926 & 0.148 & 926 & 0.148 \\
\hline Inducido & & & 1,346 & 0.215 & 1,346 & 0.215 \\
\hline Regional & & & & & 4,911 & 0.785 \\
\hline Total (ET) & 7,183 & 1.148 & 8,530 & 1.363 & 13,440 & 2.148 \\
\hline ET/VBP local & $2.6 \%$ & & $3.0 \%$ & & $4.8 \%$ & \\
\hline
\end{tabular}

Fuente: elaboración propia en base a los resultados obtenidos de las simulaciones.

A medida que el modelo complejiza su estructura añadiendo más componentes, la participación de efectos directos e indirectos se invierte, pasando los efectos directos de tener una gran importancia bajo el modelo $\mathrm{M}_{1}$ a una menor en el modelo M3.

Con respecto al multiplicador total del turismo, por ejemplo, para el modelo 3 , cada peso más gastado por los turistas genera un aumento en el valor de la producción de la localidad en más de 2 pesos.

La participación de la región en el total del impacto de valor agregado pierde importancia frente al resto de las regiones a medida que se avanza desde M1 a M3.

Tabla 6. Impacto sobre el valor agregado

\begin{tabular}{|l|c|c|c|c|c|c|}
\hline \multirow{2}{*}{ Concepto } & \multicolumn{2}{|c}{ M1 } & \multicolumn{2}{c}{ M2 } & \multicolumn{2}{c|}{ M3 } \\
\hline VAB VF-VU & ARS & Part. & ARS & Part. & ARS & Part. \\
\hline VAB Prov./Nac. & 3,225 & $76 \%$ & 3,310 & $76 \%$ & 3,311 & $45 \%$ \\
VAB total & & & & & & \\
\hline
\end{tabular}

Fuente: elaboración propia en base a los resultados obtenidos de las simulaciones.

La participación de los diferentes sectores sobre el efecto total es homogénea para cada uno de los modelos. 
Figura 3. Participación del impacto sobre las economías de VF-VU

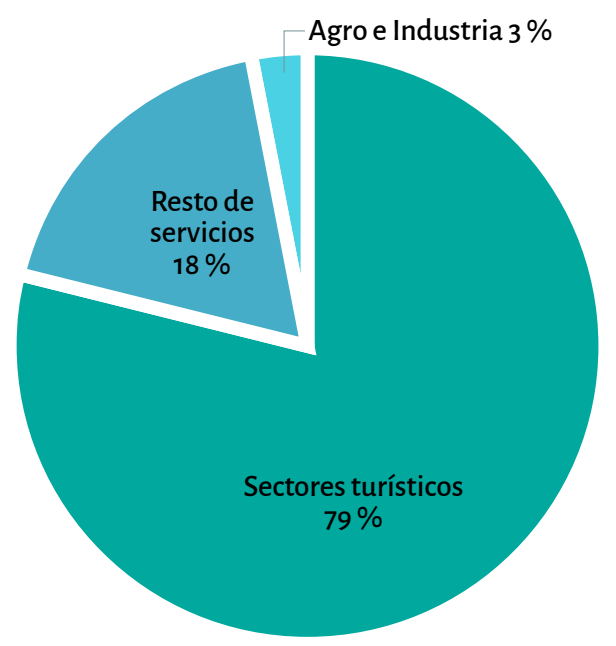

Fuente: elaboración propia en base a los resultados obtenidos de las simulaciones.

Los sectores de turismo y de industria son los que más crecen con respecto a sus valores de producción base, siendo este crecimiento de $16 \%$ y $9 \%$ respectivamente.

Desde el punto de vista del desarrollo productivo local, del análisis sectorial y, sobre todo, del derrame regional derivado del aumento del turismo, surge un interesante aspecto relacionado con las cadenas de valor. Los bajos resultados de los multiplicadores, en términos de impacto regional, parecen sugerir que habría espacio para mejorarlos. Habría que analizar si efectivamente es posible desarrollar cadenas de valor locales que permitan extender el impacto sobre la economía regional.

\subsection{Resultados del análisis costo-beneficio y de sensibilidad}

Considerando solamente el valor agregado generado en la región, el VAN tiene un valor negativo de más de 2 millones de dólares (USD) para el modelo 1 (con valores similares en los otros 2 modelos realizados). Incluso, si se considera el valor agregado generado en el total de la Argentina, se registran valores negativos de VAN para los modelos 1 y 2 , superando en ambos casos el millón de dólares. Únicamente cuando se utiliza el modelo 3 se obtiene un valor positivo superior a los 2 millones de dólares ${ }^{19}$.

Se concluye entonces que los beneficios (ex ante) recogidos dentro la región VaIle Fértil-Villa Unión no alcanzarían para recuperar los costos de inversión. Únicamente utilizando el modelo 3 , y considerando los efectos que aquella tiene sobre todo el país, la inversión otorga resultados económicamente deseables.

19 Las tasas internas de retorno (TIR) son de $8.99 \%, 9.30 \%$ y $16.43 \%$ para los modelos 1, 2 y 3 , respectivamente. 
Respecto a los resultados obtenidos a través del análisis de sensibilidad, tomando el valor agregado generado por la región en el modelo 3, es necesaria una expansión del gasto turístico superior al $16 \%$ para lograr cubrir la inversión realizada. En contraposición, considerado el efecto sobre el valor agregado de todo el país, dicho objetivo se alcanza con un incremento de $7.4 \%$.

Si bien recién a partir de expansiones superiores al $16 \%$ del gasto turístico se lograría un VAN positivo, este valor no resulta alejado del promedio de las tasas de crecimiento interanual de ingresos a cada parque. En base a datos obtenidos por el Ministerio de Turismo y Deportes de la Nación ${ }^{\mathbf{2 0}}$, los parques Talampaya e Ischigualasto mostraron, individualmente, un crecimiento interanual promedio de ingresos, entre el 2009 y el 2018 , de $20 \%$ y de $16 \%$, respectivamente.

\section{Conclusiones}

Para algunas economías regionales, los flujos provenientes del turismo pueden significar un importante impulso económico. Esto implica que la evaluación de impacto de variaciones en el turismo es de interés tanto para los responsables de implementar políticas públicas como para aquellos que las diseñan. Sin embargo, realizar investigaciones sobre el efecto del turismo a nivel regional puede enfrentar una restricción relevante: datos deficientes o inexistentes cuyos costos de obtención implicarían la erogación de gran cantidad de recursos. En este sentido, el presente trabajo evidencia el gran desafío que enfrentan regiones subnacionales, como Valle Fértil-Villa Unión, a la hora de medir el impacto de una política. No obstante, se muestra cómo, a través de las técnicas de encuestas y técnicas híbridas, es posible la construcción y estimación de tablas insumo-producto y MCS para estas regiones, que arrojan resultados confiables y de calidad, cuando no está disponible la información. Esto resulta ser un gran aporte debido a que la utilización de dichas técnicas permite la evaluación de impacto en regiones subnacionales donde antes no era posible.

A partir de las tablas insumo-producto y la MCS estimada, se utilizaron modelos de insumo-producto interregional para la cuantificación de los efectos directos e indirectos. Al aplicar dichos modelos, se encontró que una expansión en el turismo se correlaciona con el PIB regional. No obstante, a medida que se expande el modelo, incorporando efectos sobre los hogares y sobre las áreas provinciales y nacionales (M2 y M3), se ve una caída en la importancia relativa de los efectos directos respecto a los indirectos. Este hecho no tiene que ser visto como un problema, sino más bien como una virtud, donde un programa de desarrollo local contribuye en el desarrollo regional y nacional o donde un programa nacional tiene un impacto importante a

20 Ministerio de Turismo y Deporte (2020). Estadísticas de Turismo, Parques Nacionales, Informes Técnicos, http://www.yvera.tur.ar/estadistica/info/parques-nacionales/informes-tecnicos 
nivel local. Si bien estas macrorregiones capturan gran parte del impacto, una mejora en las cadenas de valor local podría permitir que la economía local y regional aprovechen más los efectos.

El análisis de costo-beneficio refuerza la idea de políticas de desarrollo con objetivos regionales y nacionales, ya que únicamente considerando el impacto del valor agregado generado en todo el país y utilizando el M3, se logra recuperar el monto de la inversión realizada y obtener beneficios positivos.

\section{Agradecimientos}

Agradecemos a las autoridades del Banco Interamericano de Desarrollo (BID) y del Ministerio de Turismo de la Nación (MINTUR), especialmente al Sr. Alejandro Roca, al Sr. Rodrigo Oliver y al Sr. Ricardo Quiroga, por su crucial ayuda durante este estudio. También agradecemos a estas instituciones el permitirnos utilizar los datos recopilados durante el desarrollo del proyecto de modelos de simulación para el monitoreo y evaluación de los impactos económicos del Programa de Desarrollo de Corredores Turísticos (préstamo del BID N²606 / OC-AR). Finalmente, el apoyo de Camila Sachella, Diego Born, Sergio Passamonti, Gustavo Vega y Conzalo Sereno fueron muy importantes en el proceso de diseño, construcción y procesamiento de diferentes encuestas de hogares y producción. Los autores son responsables de los argumentos, ideas y opiniones expresadas, las cuales no simbolizan la opinión oficial del MINTUR de Argentina ni de ninguna oficina bajo el BID.

\section{Referencias bibliográficas}

AlCOBer, O., MARtíneZ, R. \& COLOMBI, C. (2008). Parque Provincial Natural Ischigualasto. La Cuna de los Dinosaurios. Servicio Ceológico Minero Argentino. Instituto de Geología y Recursos Minerales.

ALLAN, G. J., LECCA, P. \& SWALES, K. (2017). The impacts of temporary but anticipated tourism spending: An application to the Glasgow 2014 commonwealth games. Tourism Management, 59, 325-337.

BACHARACH, M. (1970). Biproportional Matrices and Input-Output Change. Cambridge University Press.

BANCO INTERAMERICANO DE DESARROLLO (2011). Programa de desarrollo de corredores turísticos (AR-L1071): Propuesta de préstamo.

BANCO INTERAMERICANO DE DESARROLLo (2012). Programa de desarrollo de corredores turísticos (AR-L1071): Contrato de préstamo No. 2606/OC-AR.

BANERJEE, O., CICOWIEZ, M. \& CACHOT, S. (2015). A quantitative framework for assessing public investment in tourism. An application to Haiti. Tourism Management, 51, 157-173. 
Romero, C. A., TARelli, J. P. y Porto, L. (2020). El impacto económico del turismo en ... 105-128.

BOULLÓN, R. (1997). Planificación del espacio turístico. México DF: Trillas.

CHISARI, O.O., MASTRONARDI, L.]. \& ROMERO, C.A. (2012). Building an input-output Model for Buenos Aires City. MPRA paper 40028, University Library of Munich, Alemania.

COREMBERG, A., ROMERO, C.A., MASTRONARDI, L. \& VILA MARTÍNEZ, J.P. (2016). Estimación de una Matriz de Contabilidad Social para Argentina 2012: Metodología y contraste con la matriz insumo producto de 1997. LI Reunión Anual de la Asociación Argentina de Economía Política (págs. 1-26). San Miguel de Tucumán: AAEP.

DWYER, L., FORSYTH, P. \& SPURR, R. (2004). Evaluating tourism's economic effects: new and old approaches. Tourism management, 25(3), 307-317.

FLEGG, A.T., MASTRONARDI, L. J. \& ROMERO, C.A. (2015). Evaluating the FLQ and AFLQ formulae for estimating regional input coefficients: empirical evidence for the province of Córdoba, Argentina. Economic Systems Research, 28(1), 21-37 DOI:10 $.1080 / 09535314.2015 .1103703$

FLECG, A.T. \& WEBBER, C.D. (2000). Regional size, regional specialization and the FLQ formula. Regional Studies, 34(6), 563-569.

FLEGG, A.T. \& WEBBER, C.D (1997). On the Appropriate Use of Location Quotients in Cenerating Regional Input-Output Tables: Reply. Regional Studies, 31(8), 795805, DOI: $10.1080 / 713693401$

FLECG, A.T., C.D. WEBBER \& ELLIOTT, M.V. (1995) On the Appropriate Use of Location Quotients in Cenerating Regional Input-Output Tables. Regional Studies, 29, 547-561.

FRECHTLING, D.C. \& HORVÁTH, E. (1999). Estimating the multiplier effects of tourism expenditures on a local economy through a regional input-output model. Journal of travel research, 37(4), 324-332.

GREENSTREET, D. (1989). A Conceptual Framework for Construction of Hybrid Regional Input-Output Models. Socio-Economic Planning Sciences, 23(5), 283289.

INDEC (2018). Balanza de pagos, posición de inversión internacional y deuda externa. -Recuperado de: https://www.indec.gob.ar/indec/web/Nivel4-Tema-3-35-45

JENSEN, R.C., MANDEVILLE, T.D. \& KARUNARATNE, N.D. (1979). Regional economic planning. Croom Helm Series in Ceography and Environment.

MACROCONSULTING (2016). Elaboración de una línea de base y desarrollo de los modelos de simulación para el programa de desarrollo de corredores turísticos. Programa de Desarrollo de Corredores Turísticos, Ministerio de Turismo-BID, septiembre.

MASTRONARDI, L. (2015). Estimación indirecta e híbrida de matrices insumo producto regionales bajo diferentes criterios informativos. Un análisis de precisión al cambiar el tamaño de región. L Reunión Anual de la Asociación Argentina de Economía Política, Salta, Argentina. Volume: 50. 10.13140/RG .2.1.1856.6164. 
Romero, C. A., TARelli, J. P. y Porto, L. (2020). El impacto económico del turismo en ... 105-128.

MASTRONARDI, L.J. \& ROMERO, C.A. (2012). Estimación de matrices de insumo producto regionales mediante métodos indirectos. Una aplicación para la ciudad de Buenos Aires. Documento de trabajo, MPRA paper 37006, University Library of Munich, Alemania.

MCDOUGALL, R.A. (1999). Entropy theory and RAS are friends. GTAP Working Papers, 6.

MILLER, R.E. \& BLAIR, P.D. (2009). Input-output analysis: foundations and extensions. Cambridge University Press.

MINISTERIO DE TURISMO DE LA NACIÓN, ANUARIO ESTADÍSTICO DE TURISMO (2015). Recuperado de: https://www.yvera.tur.ar/estadistica/documentos/ descarga/59foo $80 a 467 \mathrm{fb} . p d f$

MINISTERIO DE TURISMO Y DEPORTE (2020). Estadísticas de Turismo, Parques Nacionales, Informes Técnicos. Recuperado de: http://www.yvera.tur.ar/ estadistica/info/parques-nacionales/informes-tecnicos

MITROVIC, M. (2019). Valle Fértil - Talampaya y Valle de la Luna (Ischigualasto). Recuperado de: https://happyfrogtravels.com/es/valle-fertil-talampaya-eischigualasto/

OOSTERHAVEN, J. \& FAN, T. (2006). Impact of international tourism on the Chinese economy. International Journal of Tourism Research, 8(5), 347-354.

RARIZA (2015). Estudio de Impacto Ambiental: Reordenamiento de la recepción del sector ingreso al Parque Provincial Ischigualasto, San Juan, Corredor Villa Unión-Valle Fértil (Región Cuyo). Recuperado de: http://turismo.gov.ar/sites/default/files/ evaluacion_de_impacto_ambiental.pdf

ROBINSON, S., CATTANEO, A. \& EL-SAID, M. (2001). Updating and Estimating a Social Accounting Matrix Using Cross Entropy Methods. Economic Systems Research 13(1), 47-64.

ROMERO, C.A., MASTRONARDI, L.J., TARELLI, J.P. \& HASLOP, F. (2019). The Regional Impact of Tourism when Data is Scarce. An Application to the Province of Salta. Tourism Planning and Development, 1-17. DOI: 10.1080/21568316.2019.1673808

SALVADEO, V. \& RUARTE, P. (2018). Patrimonio mundial: Parque Nacional Talampaya - Importancia en la gestión turística. Serie Correlación Ceológica, 34(2), 80-89.

SECCHI, L.O. (2016). Plan de Comercialización para el Parque Nacional Talampaya año 2017/2018 (tesis de grado). Universidad Siglo 21.

SECRETARÍA DE TURISMO, PRESIDENCIA DE LA NACIÓN (2018). Encuesta de Viajes y Turismo de los Hogares: Turismo interno: Cuarto Trimestre de 2018. Recuperado de: https://www.yvera.tur.ar/estadistica/documentos/ descarga/5ccoboa57c4b5559056777.pdf

SINCLAIR, M.T. (1998). Tourism and economic development: A survey. The journal of development studies, 34(5), 1-51.

SZABÓ, N. (2015). Methods for regionalizing input-output tables. Regional Statistics, 5(1), 44-65. DOI: 10.15196/RSO5103 
Romero, C. A., TARelli, J. P. y Porto, L. (2020). El impacto económico del turismo en ... 105-128.

TAYLOR, J.E. (2010). Technical guidelines for evaluating the impacts of tourism using simulation models. Impact Evaluation Guidelines. Technical Notes, No IDB-TN-229.

TAYLOR, J.E., HARDNER, J. \& STEWART, M. (2009). Ecotourism and economic growth in the Galapagos: an island economy-wide analysis. Environment and Development Economics, 14(2), 139-162.

TAYLOR, J.E., DYER, G.A., STEWART, M., YUNEZ-NAUDE, A. \& ARDILA, S. (2003). The economics of ecotourism: A Galápagos Islands economy-wide perspective. Economic Development and Cultural Change, 51 (4), 977-997.

UNESCO (s/f). Ischigualasto / Talampaya Natural Parks. Recuperado de: https://whc. unesco.org/en/list/966

VERGARA, M. (2012). Plan de Uso Público Parque Provincial Ischigualasto-Provincia de San Juan (tesis doctoral). Universidad Siglo XXI.

WORLD TOURISM ORGANIZATION (2020). Country Profile - Inbound Tourism. Recuperado en enero de 2020, de: https://www.unwto.org/country-profileinbound-tourism 